\title{
Quantitative regional curvature analysis: validation in animals of a method for assessing regional ventricular remodelling in ischemic heart disease
}

\author{
G.B. John Mancini \& Mark J. McGillem \\ Department of Internal Medicine, University of Michigan Medical School, Veterans Administration and \\ University of Michigan Medical Centers, Ann Arbor, Michigan, USA
}

Accepted 30 July 1991

Key words: shape analysis, regional function, wall motion, wall thickening, ventricular function, ischemia, coronary disease, remodelling

\begin{abstract}
Recent studies show the impact of left ventricular shape and remodelling on patient prognosis. This mandates the development of quantitative methods for measuring shape. Quantitative regional curvature analysis (QRCA) was developed to quantitate shape on a regional basis so that measurements would not be constrained to assessment of only global shape and would, therefore be applicable to ischemic heart disease. To validate QRCA, eleven dogs were instrumented with coronary occluders and radiopaque markers on the epicardium and endocardium to provide fiducial points for calculation of shape, motion and thickening. These parameters were measured in the anterior and inferior walls, at rest, during left anterior descending occlusion and finally during circumflex occlusion. QRCA showed increased curvature (increased globularity) in each wall when thickening and motion deteriorated during occlusion. The most marked shape changes occurred in the inferior wall whereas the most marked deterioration of function was detected by wall thickening measurements of the anterior wall. Thus, QRCA detects regional ventricular shape disorders coincident with regional dysfunction induced by ischemia. These changes show regional heterogeneity and demonstrate the potential importance of this measurement as opposed to simple, global measures of shape. QRCA is, therefore, suitable for monitoring acute changes of shape that occur during acute ischemia.
\end{abstract}

\section{Introduction}

Shape and remodelling are increasingly being recognized as important parameters in the assessment of ventricular function [1]. Quantitative Regional Curvature Analysis (QRCA) was specifically developed to provide quantitative measures of these important attributes and processes that characterize the ventricular response to injury [2-7]. Moreover, it was developed to allow measurements of ventricular shape on a regional basis that are independent of ventricular size, or volume. QRCA has been applied to clinical studies of contrast ventricu- lography which have shown that parameters of shape correlate with measures of regional wall motion [4-5]. However, due to uncertainties in the location of the endocardial wall in contrast ventriculographic studies, these applications cannot be considered suitable for validating the method or determining whether shape changes parallel acute deterioration of regional function. Therefore, the purpose of the study was to validate QRCA in animals with implanted, lead markers that provide a fiducial reference for ventricular wall motion and wall thickening measurements and to determine if conformational changes measured by QRCA par- 


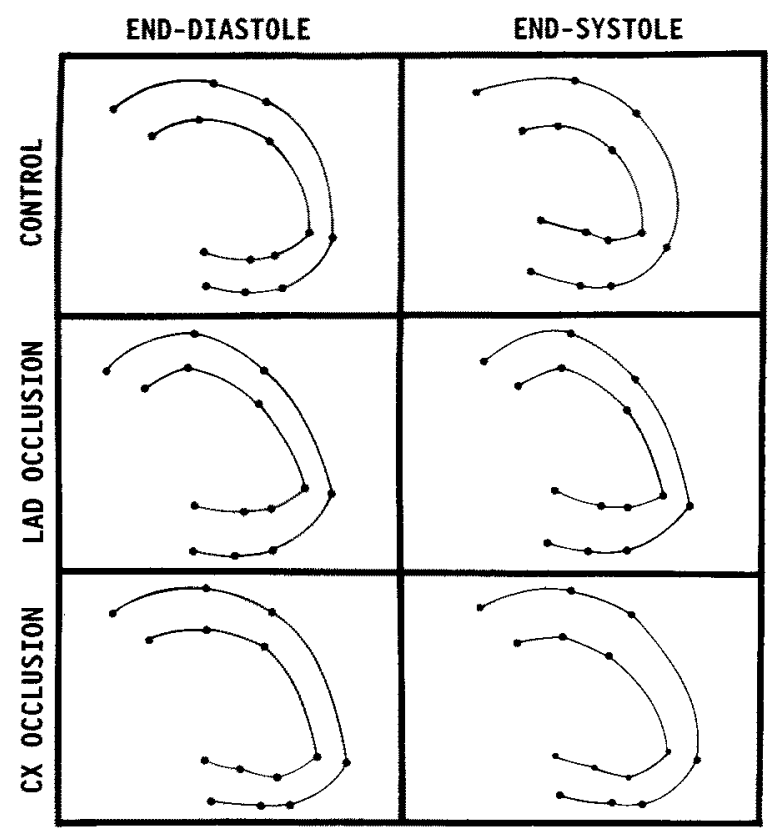

Fig. 1. The position of the epicardial and endocardial lead markers were traced from the videotaped fluoroscopic images. Their locations at end-diastole and end-systole are shown by the dots joined by lines constructed with a French curve. A set of tracings from one animal is shown for control (upper panel), left anterior descending occlusion (middle panel) and circumflex coronary occlusion (bottom panel).

alleled acute, regional, ischemic dysfunction. These shape changes are the precusors to longterm, structural remodelling of the ventricle.

\section{Methods}

Eleven conditioned dogs weighing approximately $24 \mathrm{~kg}$ on average were anesthetized with sodium pentobarbital $(35 \mathrm{mg} / \mathrm{kg})$, ventilated and subjected to a left thoracotomy. The pericardium was opened and the heart suspended in a pericardial cradle. A high fidelity micromanometer (5 French) (Millar Instruments, Houston TX) was inserted through a carotid arteriotomy into the ascending aorta to monitor central arterial pressure. The EKG was continuously monitored. The proximal left anterior descending and circumflex arteries were dissected and encircled with a snare occluder. Seven pairs of lead markers were imbedded into the myo- cardium. One of each pair was embedded in the subendocardium adjacent to the endocardium and the other was sutured on the epicardium. Three pairs were implanted in the anterior wall, paralleling the left anterior descending coronary artery and three were implanted in the inferior wall, paralleling the posterior descending coronary artery. The last pair was imbedded at the apex. A fluoroscopic x-ray unit (Orbitor, Picker International, Inc., Cleveland, Ohio) equipped with a super VHS video recorder (Panasonic Model AG-7500A, Secaucus, New Jersey) was positioned in the left posterior oblique position and oriented to maximize separation of the paired beads, the apparent endocardial cavity outline, apparent wall motion and wall thickening.

Fluoroscopic video recordings were made during a control state and after a 5 minute, total occlusion of the left anterior descending and the circumflex arteries. Sufficient time (minimum of 15 minutes) was allowed between coronary occlusions to allow for recovery of control hemodynamic conditions as well as wall motion and thickening. During occlusions, the heart rate and left ventricular end-diastolic pressures showed expected increases that resolved after resolution of the ischemia. Systolic blood pressure showed little change during the relatively brief occlusions.

The position of the lead markers at end-diastole and end-systole were traced onto a clear acetate and reoriented to mimic a right anterior oblique ventriculogram by simply turing the acetate over so that we were able to work with the mirror image of the original left anterior oblique projection (Fig. 1). The lead markers were traced with respect to fixed positions of the ribs and diaphragm (fixed, external reference system). Lines were drawn between epicardial and endocardial markers using a French curve to produce continuous epicardial and endocardial borders. These tracings were photographed using $35 \mathrm{~mm}$ film. The film images were then digitized using a Vanguard Cineviewer interfaced to an ADAC 4100C Digital Image Processing Computer (Milpitas, California) and subjected to quantitative analysis.

QRCA is based on standard vector differentiation formulae used to calculate curvature of any arc 
in space and the algorithm has been described in detail elsewhere [2-7]. Briefly, the degree of curvature (equalling the reciprocal of the radius of curvature) is calculated at 100 points around the perimeter of the ventricular silhouette, commencing at the anterior aspect of the aortic valve plane and proceeding to the junction of the mitral valve plane and the posterior aspect of the aortic valve plane. In this study, these analyses proceeded from the first lead marker placed in the anterobasal area and proceeded to the last lead marker placed in the posterobasal area. The curvature measurement simply reflects the degree of roundedness or globularity of a specified segment or arc length. High curvature values reflect highly rounded segments such as might be seen in an aneurysm or at the apex of a ventricular silhouette. Low curvature values suggest that the segment is relatively straight or only mildly curved. The latter is typical of the normal anterior wall. Negative curvature values signify that the segment is curved inward with respect to the center of the ventricular silhouette. This is sometimes seen in the inferior wall, especially in human ventriculograms. In this study, the anterior segment was defined from the anterobasal to the apical lead marker (spanning 4 lead markers) and the inferior segment was defined from the apical lead marker to the posterobasal marker (also spanning 4 lead markers). The curvature values (having units of length ${ }^{-1}$ ) are normalized by multiplying the value by the length of the ventricular silhouette. This process renders the result dimensionless and independent of size or volume. In both chronic and acute ischemia, the end-diastolic shape changes relatively little whereas end-systolic shape shows characteristic increases in curvature and globularity [3]. Therefore, the curvature results reported in this study are the end-systolic values.

Wall motion was assessed using the previously described centerline method $[8,9]$. Briefly, a certerline is constructed between the end-diastolic and end-systolic outlines, 100 equidistant chords are constructed perpendicular to it and the length of these chords is divided by the end-diastolic perimeter length to yield a fractional shortening measurement. Anterior and inferior motion were measured from the anterobasal marker to the apical
340CONTROL
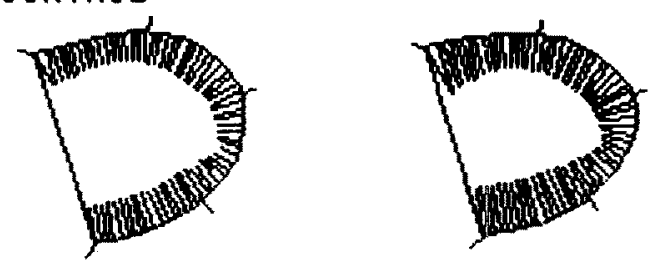

348LADOCCL
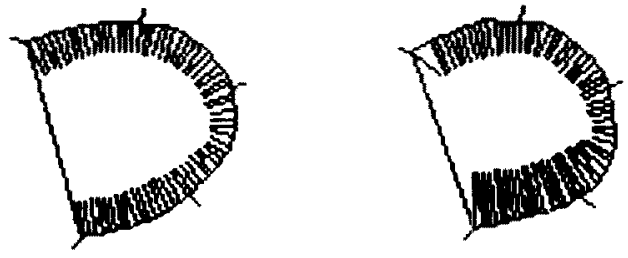

$340 \mathrm{C} \times O \mathrm{OCL}$
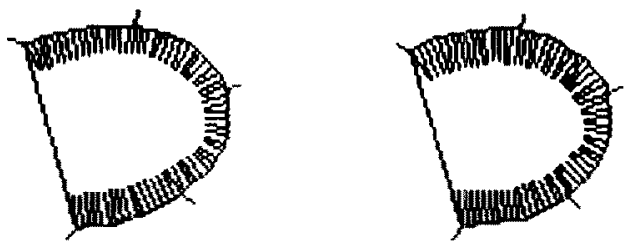

Fig. 2. A set of images showing the chords used for calculating wall thickening is shown in the same format as Figure 1.

marker and the apical marker to the posterobasal marker as described above. Results were expressed as the mean fractional shortening in the defined regions (i.e. mean of each chord length divided by the end-diastolic perimeter length).

Wall thickness was assessed using a previously developed and validated modification of the centerline wall motion method $[10,11]$. In this program, 100 perpendicular chords are drawn to the centerline between the epicardial and endocardial edges of end-diastolic and end-systolic images. The mean length of these chords in the anterior and inferior zones were used to calculate percent wall thickening:

$$
\mathrm{WT}(\%)=\left[\left(\mathrm{wt}_{\mathrm{es}}-\mathrm{wt}_{\mathrm{ed}}\right) / \mathrm{wt}_{\mathrm{ed}}\right] \times 100 \%
$$

where WT (\%) is percent wall thickening, wt is mean wall thickness, ed is end-diastole and es is end-systole. The mean thickening values were determined from the identical regions defined for measures of anterior and inferior shape and mo- 

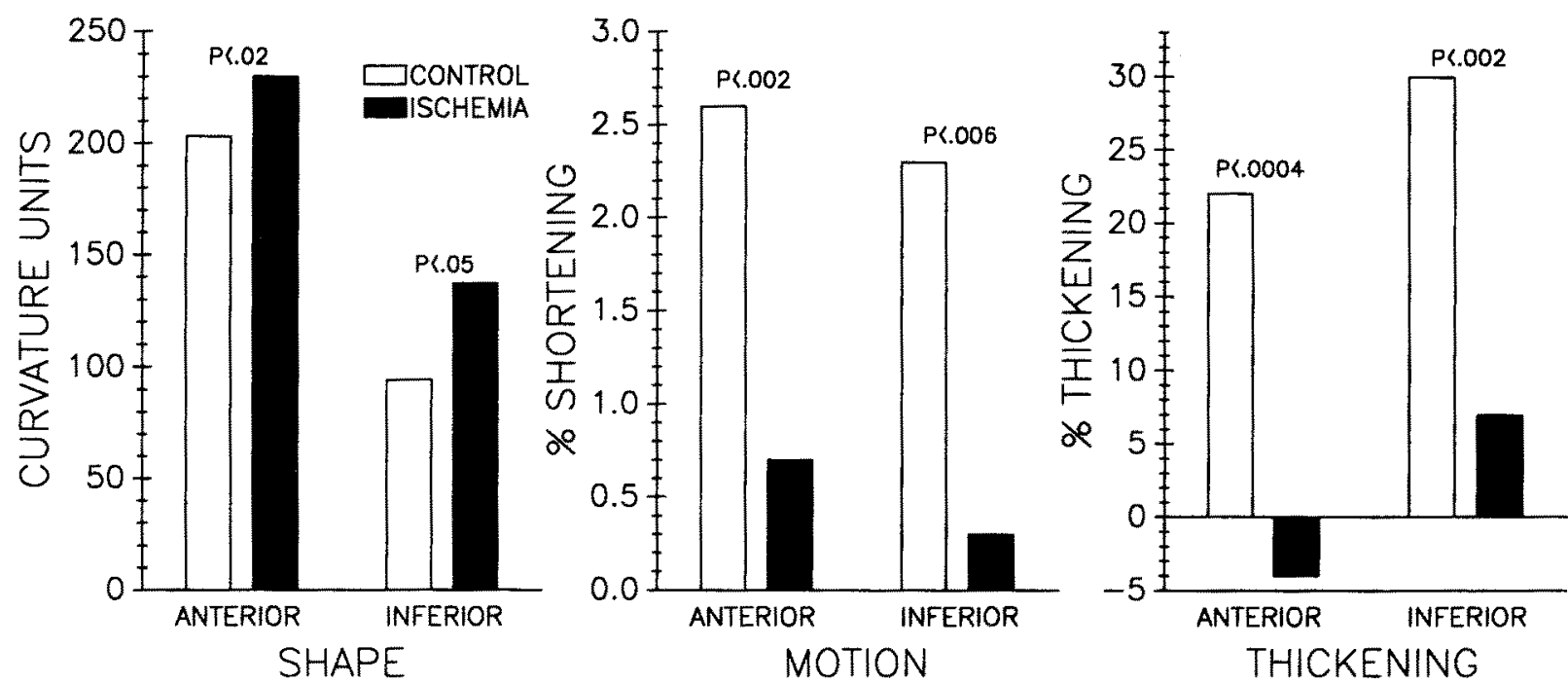

Fig. 3. Results of shape, motion and thickening analyses are demonstrated for the anterior and inferior walls during control and ischemic conditions.

tion. Figure 2 shows a composite of the display of a series of images and the wall thickness chords for a single animal studied in the control state, during left anterior descending coronary artery occlusion and finally, during left circumflex coronary artery occlusion.

Directional changes in each parameter of regional function were assessed by a paired Student's $\mathrm{t}$-test and considered significant if $\mathrm{p}<0.05$.

\section{Results}

Figure 3 shows the results of the directional changes in shape, motion and thickening in each vascular territory. During acute ischemia, the gently curved anterior wall becomes more curved and bulges outward significantly $(203 \pm 54$ vs $230 \pm 56, \mathrm{p}<0.02$, mean \pm 1 standard deviation, unitless curvature units). This represents a mean percentage change of $13 \%$. The inferior wall is less curved than the anterior wall during baseline conditions but shows a relatively greater proportionate increase in curvature during ischemia than the anterior wall ( $94 \pm$ 31 vs $137 \pm 64, p<0.05$ ). This is a mean change of $46 \%$.

These conformational changes occur in associ- ation with the expected deterioration in both motion and thickening. Wall motion fell from $2.6 \pm$ $1.2 \%$ to $0.7 \pm 0.5 \%$ in the anterior wall $(\mathrm{p}<$ 0.002 ) and from $2.3 \pm 0.7$ to $0.3 \pm 0.7 \%$ in the inferior wall $(\mathrm{p}<0.006)$. The basal level of wall motion and the extent of deterioration were relatively comparable in both walls and represent mean changes of $75 \%$ (anterior wall) and $85 \%$ (inferior wall). These directional changes were larger than the percentage changes in shape.

Wall thickening changes were the most dramatic in the anterior wall. The anterior wall thickening deteriorated from $22 \pm 10 \%$ to $-4 \pm 12 \%$ (i.e. net wall thinning was detected) $(\mathrm{p}<0.0004)$. The inferior wall thickening deteriorated from $30 \pm$ $14 \%$ to $7 \pm 9 \% \quad(p<0.002)$. These represent mean, percent changes of $118 \%$ and $77 \%$, respectively.

\section{Discussion}

Acute ischemia causes the rapid onset of conformational changes in each ischemic wall. These conformational changes can be detected by Quantitative Regional Curvature Analysis based on tracking of fiducial, lead markers imbedded in the myocardi- 
um. These shape changes are in the same general direction as the expected long-term consequences of a permanent total occlusion with infarction and eventual healing. Thus, these early functional changes can be considered to be the precursors to long-term, structural remodelling of the ventricle. These results are not affected by theoretical difficulties of measuring shape from contrast ventriculograms wherein tracking of particular segments of the myocardium may be unclear and trabecular invaginations may not accurately reflect endocardial edge definition. Nevertheless, these results are entirely consistent with the directional changes measurable from contrast studies.

The shape changes detected in this study occurred in parallel with reductions in regional wall motion and wall thickening. Regional heterogeneity in these responses were noted and this also was a function of the method of measurement. That is, although acute ischemic regional dysfunction as assessed by wall motion analysis was relatively homogeneous, the shape changes and the wall thickening changes showed opposite effects in the different regions. The anterior wall showed the most pronounced deterioration of wall thickening. This consisted of a $118 \%$ decrease due to net wall thinning. But this was accompanied by only a $13 \%$ increase in regional curvature. In contrast, the $77 \%$ reduction in inferior wall thickening, which was more modest than noted in the anterior wall, was accompanied by a larger change in shape $(46 \%$ change) than that which occurred in the anterior wall. These findings result most likely from the marked disparity between anterior and inferior wall shape in the basal state, since the inferior wall is much flatter and, therefore, subject to potentially higher regional wall stress than the anterior wall.

The heterogeneity of response to shape provides an important justification for considering regional measurements in studies of ventricular remodelling in ischemic heart disease. That is, just as the overall ejection fraction may not adequately reflect deranged ventricular function in the presence of regional ischemic dysfunction and contralateral compensation, so too might exclusive reliance on global shape parameters fail to demonstrate clear conformational changes that occur on a regional basis.
In conclusion, this study shows that the conformational changes occurring in acute ischemia are generally more subtle than changes in wall motion and wall thickening and that these changes demonstrate regional heterogeneity, especially with regard to shape and thickening changes. This heterogeneity is likely to be a direct effect of the disparity in the initial shapes and resultant stresses of the anterior and inferior walls. These early functional changes in shape are consistent in form to the longterm, structural changes that are seen during the healing of myocardial infarctions. Thus, the Quantitative Regional Curvature Analysis algorithm is suitable for detecting these subtle, acute changes and is, therefore, a suitable method for quantitatively monitoring the remodelling process from its onset in ischemic heart disease.

\section{Acknowledgement}

This study was supported by The National Institute of Health, Bethesda, Maryland, RO1-HL36813.

\section{References}

1. Pfeffer MA, Braunwald E. Ventricular remodeling after myocardial infarction: experimental observations and clinical implications. Circulation 1990; 81: 1161-72.

2. Mancini GBJ, LeFree MT, Vogel RA. Curvature analysis of normal ventriculograms: fundamental framework for the assessment of shape changes in man. Comput Cardiol 1985; $141-4$

3. Mancini GBJ, DeBoe SF, Anselmo E, Simon SB, LeFree MT, Vogel RA. Quantitative regional curvature analysis: an application of shape determination for the assessment of segmental function in man. Am Heart J 1987; 113: 326-34.

4. Mancini GBJ, DeBoe SF, Anselmo E, LeFree MT. A comparison of traditional wall motion assessment and quantitative shape analysis: a new method for characterizing left ventricular function in humans. Am Heart J 1987; 114: 1183-91.

5. Mancini GBJ, DeBoe SF, McGillem MJ, Bates ER. Quantitative regional curvature analysis: a prospective evaluation of ventricular shape and wall motion measurements. Am Heart J 1988; 116: 1616-21.

6. Mancini GBJ, Starling MR. Heterogeneity of regional wall motion and shape in response to altered load (abstract). $J$ Am Coll Cardiol 1989; 13:101A.

7. Mancini GBJ, Scott F, DeBoe SF, Gillon J, Simon SB, 
LeFree MT, Weymouth TE. Measurement of systolic and diastolic disorders of shape using frame-by-frame quantitative regional curvature analysis. Cor Art Dis 1991; 2: 17987.

8. Sheehan FH, Dodge HT, Mathey DG, Bolson EL, Mitten S. Application of the centerline method: analysis of change in regional left ventricular wall motion in serial studies. Comput Cardiol 1980: 97.

9. Bolson EL, Kilman S, Sheehan FH, Dodge HT. Left ventricular segmental wall motion: a new method using local direction information. Comput Cardiol 1980: 245.

10. McGillem MJ, Mancini GBJ, DeBoe SF, Buda AJ, Mod- ification of the centerline method for assessment of echocardiographic wall thickening and motion: a comparison with areas of risk. J Am Coll Cardiol 1988; 11: 861-6.

11. McGillem MJ, Pinto IMF, Mancini GBJ. Determination of wall thickening and myocardial mass by digital ventriculography. Am J Card Imag 1989; 3: 244-52.

Address for offprints:

G.B. John Mancini, M.D., V.A.M.C. (111A),

2215 Fuller Road,

Ann Arbor, Michigan 48105, USA 